\title{
Ultradrawing of Isotactic Polypropylene Films Produced by Gelation/Crystallization from Solutions
}

\author{
Masaru Matsuo, ${ }^{*}$ Chie Sawatari, and Toshi NAKano \\ Department of Clothing Science, Faculty of Home Economics, \\ Nara Women's University, Nara 630, Japan
}

(Received March 25, 1986)

\begin{abstract}
Films of ultra-high molecular weight isotactic polypropylene $\left(4.4 \times 10^{6}\right)$ were produced by gelation/crystallization from dilute solutions according to the method of Smith and Lemstra. The dried gel films could be readily stretched to a maximum draw ratio of 100 in a hot oven at $165-170^{\circ} \mathrm{C}$. For a draw ratio of 100 , tensile strength and the Young's modulus attained the maximum values of 1.56 and $40.4 \mathrm{GPa}$, respectively, at $20^{\circ} \mathrm{C}$. These values are among the highest reported for polypropylene. The tensile strength was beyond the theoretical value reported by Samuels and the Young's modulus is close to the theoretical value measured by X-ray diffraction. KEY WORDS Ultra-High Molecular Weight Polypropylene / Dried Gel Film / Tensile Strength / Young's Modulus /
\end{abstract}

In recent years, research on the preparation of fibers and films with high modulus and high strength has become a topic of increasing interest and this problem has been concentrated on polyethylene. ${ }^{1-11}$ In attempt to produce such fibers (films), gel deformation is one of excellent methods. This method was first proposed by Smith et al. ${ }^{5-6}$ Gels were prepared by gelation/crystallization from dilute solutions of ultra-high molecular weight polyethylene and decalin used as the solvent was allowed to evaporate from the gels under ambient conditions. The dry gels were elongated at $130-135^{\circ} \mathrm{C}$. Recently, Matsuo and Sawatari succeeded in producing ultradrawn films whose Young's modulus attained $216 \mathrm{GPa}$ corresponding to almost theoretical values measured by $\mathrm{X}$-ray diffraction technique. ${ }^{11}$ The drawn ratio attained to about 400 times and the second order orientational factor of the crystal $c$-axes was 0.99999 indicating almost complete orienta- tion in the stretching direction.

The origin of such a consistently high draw ratio was attributed to a reduced number of entanglements per molecule in solution-cast/ spun polymer in comparison with those obtained from the melt. Based on the above concept for polyethylene, Peguy and Manley ${ }^{12}$ applied the method to the ultradrawing of polypropylene which belongs to poly- $\alpha$-olefine groups such as polyethylene. According to their report, ${ }^{12}$ the dried gel films were prepared by using a similar method to the preparation of polyethylene gel films. The dried gel films were stretched in the temperature range from 110 to $160^{\circ} \mathrm{C}$, up to draw ratio of $\lambda=57$. The Young's modulus and tensile strength were 36 and $1.05 \mathrm{GPa}$, respectively, at room temperature. From another aspect, Kanamoto et al. studied extrusion of single crystal mats of polypropylene. They pointed out that the Young's modulus and tensile strength attained maximum values of 30 and

* To whom all correspondence should be addressed. 


\section{$1.5 \mathrm{GPa}$, respectively. ${ }^{13}$}

In this paper, the origin of ultradrawing of polypropylene is discussed in terms of a suitable level of entanglements. This problem has been approached by measurement of solution viscosity, since it is basically related to the size or extension in space of macromolecules.

Secondly, the morphological and thermal properties of the dried gel films were investigated by using small-angle X-ray scattering (SAXS), wide angle $\mathrm{X}$-ray diffraction (WAXD), polarized microscopy, crystallinity, and differential scanning calorimetry (DSC).

Finally, the mechanical properties are discussed as a function of draw ratio. Measurements were carried out with a tensile tester at room temperature and with a viscoelastic spectrometer over the temperature range from -150 to $180^{\circ} \mathrm{C}$ at a fixed frequency of $10 \mathrm{~Hz}$.

\section{EXPERIMENTAL}

Isotactic polypropylene with high molecular weight $\left(\bar{M}_{v}=4.4 \times 10^{6}\right)$ was used as a specimen. The solvent was decalin. For viscosity measurements, the dissolution was carried out at three temperatures, 135, 150, and 170 ${ }^{\circ} \mathrm{C}$ under nitrogen and the solutions were stabilized with $0.1 \% \mathrm{w} / \mathrm{w}$ of antioxidant (di- $t$ butyl-p-cresol). The viscosities of the solutions were determined by capillary viscometry at these three temperatures.

Gel films were prepared by crystallization from solutions. Decalin solutions containing the approximate amount of polypropylene and $0.1 \% \mathrm{w} / \mathrm{w}$ of the antioxidant di- $t$-butyl- $p$ cresol were prepared by heating the wellblended polymer/solvent mixture at three temperatures for $40 \mathrm{~min}$ under nitrogen. The hot homogenized solution was quenched by pouring into an aluminum tray which was surrounded by ice water, thus generating a gel. The decalin was allowed to evaporate from the gel under ambient conditions. The nearly dried gel film was immersed in an excess of ethanol and subsequently air-dried to remove residual traces of the decalin-ethanol mixture. The dried gel film was cut into strips of length beyond $35 \mathrm{~mm}$ and width $10-15$ $\mathrm{mm}$. The strips were clamped into a manual stretching device in such a way that the length to be drawn was $15 \mathrm{~mm}$. The specimens were placed in an oven at $165-170^{\circ} \mathrm{C}$ and elongated manually to the desired draw ratio below $\lambda=20$. Immediately after stretching, the stretcher with the sample was quenched to room temperature. Draw ratios were determined in the usual way by measuring the displacement of ink marks placed $2 \mathrm{~mm}$ apart on the specimen prior to drawing. The elongation up to higher draw ratios beyond $\lambda=$ 20 was done in a second stage. This was due to the difficulty in obtaining drawn films beyond $\lambda=20$ in one step because of the physical restriction of the size of the oven. Thus the specimen with an original length was drawn to $\lambda=20$ in the first stage and the drawn film was cut into strips of length $50-60 \mathrm{~mm}$. These specimens, each clamped over a length of $10 \mathrm{~mm}$ at the ends, the length to be stretched being $30-40 \mathrm{~mm}$, were drawn to the desired ratio beyond $\lambda=20$, in the second stage using the method described above.

The mechanical properties of the drawn films were measured with a tensile tester at a room temperature and with a viscoelastic spectrometer (VES-F) of Iwamoto Machine Co., Ltd. over the temperature range from -150 to $180^{\circ} \mathrm{C}$ at $10 \mathrm{~Hz}$.

The X-ray measurements were carried out by $12 \mathrm{~kW}$ rotating anode $\mathrm{X}$-ray generator (RDA-rA). WAXD patterns were obtained with a flat film camera using $\mathrm{Cu}-K_{\alpha}$ radiation at $200 \mathrm{~mA}$ and $40 \mathrm{kV}$. The X-ray beam was monochromatized by a curved graphite monochrometer. The exposure time for all specimens was $30 \mathrm{~min}$, when the incident beam was directed parallel to the film surface (end view). The exposure time was $1 \mathrm{~h}$, when the incident beam was along the film normal 
direction (through view).

SAXS patterns were obtained with a flat film camera. The exposure time for all the specimens was $100 \mathrm{~h}$ both in the case of through and end views.

SAXS intensity distribution in the meridional direction was detected with a position sensitive proportional counter (PSPC) at $200 \mathrm{~mA}$ and $40 \mathrm{kV}$. The measurement was carried out with point focussing in a threeslit system. The corrected intensity was obtained by substracting the contribution of the background (corresponding to air scattering) from total intensity. The $\mathrm{X}$-ray exposure time was $4 \times 10^{5} \mathrm{~s}$ each for total and air scatterings.

Scanning electron and polarized micrographs were obtained with a JSM-T300 and a Nikon HFM, respectively.

Light scattering patterns were obtained with a 3-mW He-Ne gas laser as a light source. Diffuse surfaces were avoided by sandwitching the specimen between microcover glasses with silicon oil (refraction index 1.556) as an immersion fluid.
The fusion behavior of drawn specimens was studied by differential scanning calorimetry (DSC) using a Rigaku Denki calorimeter (8089 TG-DSC). Each film was cut into square pieces weighting about $5 \mathrm{mg}$ and the samples were placed in standard aluminum sample pans. Samples were heated at a constant rate of $10 \mathrm{~K} \mathrm{~min}^{-1}$. Since the shape of the DSC traces and the location of the peak for a given heating rate are very dependent on the packing, geometry and size of the specimens, these parameters were kept as constant as possible.

Birefringence measurements were made in a polarizing microscope using a Babinet compensator for measuring the retardations.

Film density was measured by a pycnometer with chlorobenzene-toluene as the medium. A great care was taken to remove the antioxidant, since the density is remarkably affected by the residual antioxidant. Then the drawn specimen was cut into fragments and they were immersed in an excess of ethanol for more than 30 days prior to measuring the density and subsequently vacuum-dried for 3

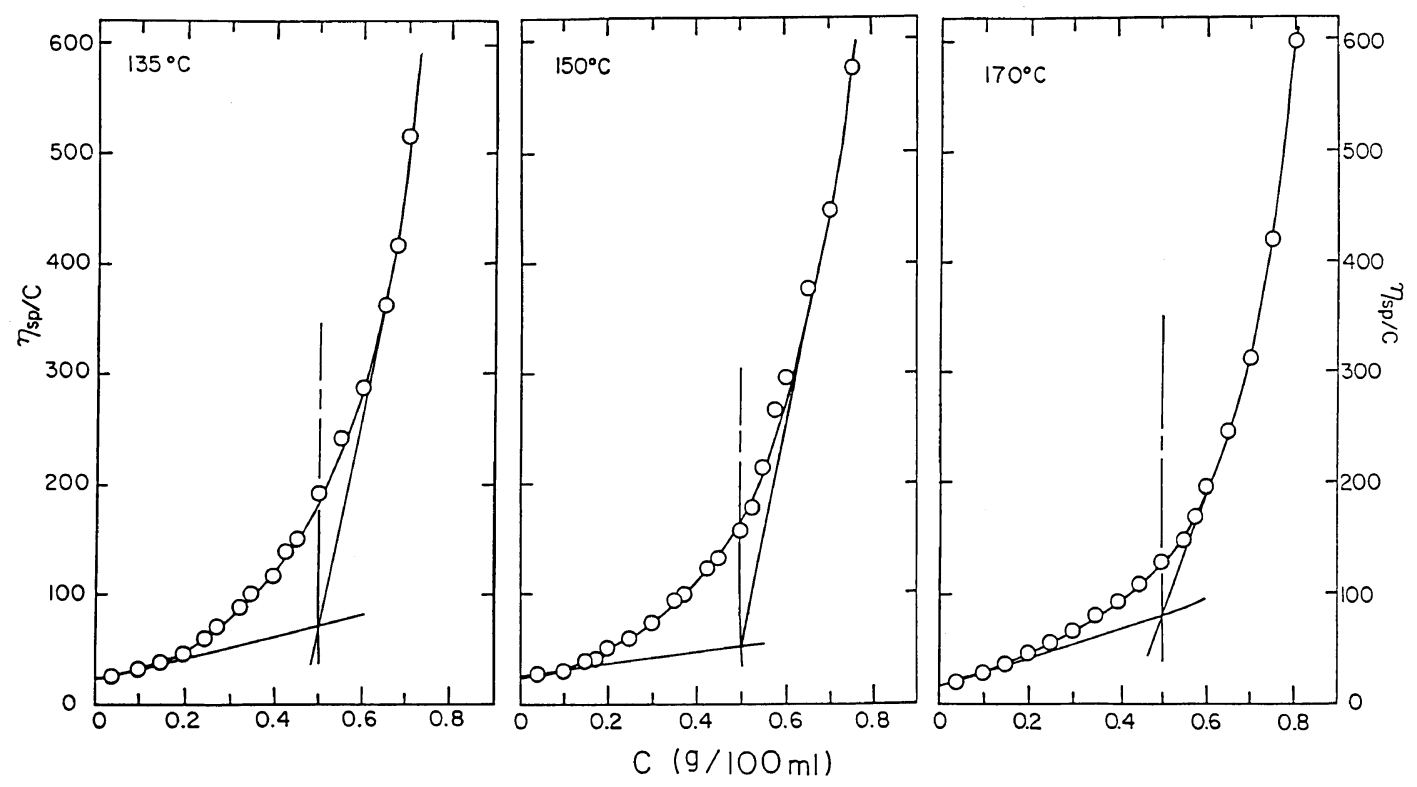

Figure 1. Relationship between reduced viscosity $\eta_{\mathrm{sp}} / c$ and concentration $c$ for solutions of high molecular weight polypropylene in decalin. 


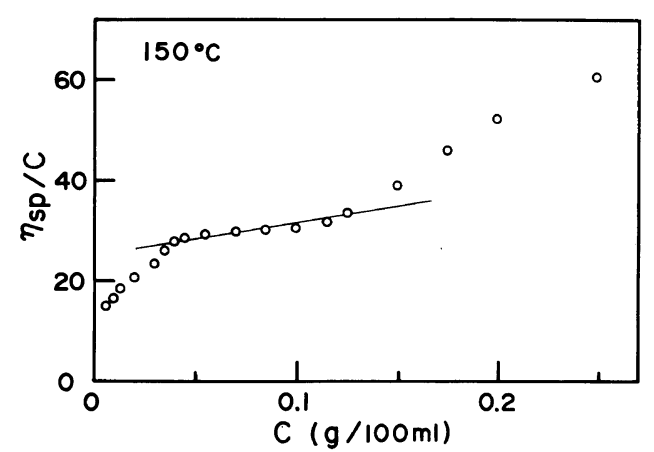

Figure 2. Enlargement of Fig. 1 in the low concentration regime.

days. Crystallinity was calculated assuming the crystal and amorphous densities to be 0.938 and $0.85 \mathrm{~g} \mathrm{~cm}^{-3}$, respectively. ${ }^{14}$

\section{RESULTS AND DISCUSSION}

Figure 1 shows the whole profile of the reduced viscosity-concentration relationship. This relationship is hardly affected by the temperature of dissolution in the range from 135 to $150^{\circ} \mathrm{C}$. The viscosity measurement pointed out that $135^{\circ} \mathrm{C}$ is not a suitable temperature for effective dissolution and at 170 ${ }^{\circ} \mathrm{C}$, it is unavoidable that degradation of molecular weight occures. The viscosity measurement supports the fact that $150^{\circ} \mathrm{C}$ is the best temperature for effective dissolution without degradation.

Figure 2 shows a detailed profile of the reduced viscosity-concentration relationship in the concentration regime lower than $0.25 \mathrm{~g} / 100 \mathrm{ml}$ at the dissolution temperature of $150^{\circ} \mathrm{C}$. In this low concentration regime, the relationship can be represented as three straight lines, respectively.

The critical concentration for coil overlap can be estimated from the relationship $[\eta] c^{*}=1,{ }^{15}$ where $[\eta]$ is the intrinsic viscosity. For the present system, $[\eta]=17 \mathrm{dl} \mathrm{g}^{-1}$ and thus the value $c^{*}$ is about $0.07 \mathrm{~g} \mathrm{dl}^{-1}$. This is far below the concentration whose optimum draw characteristics are obtained. This empirical observation implies that the critical concentration determined in this way is not correlated with optimum draw characteristics. Furthermore, there is an internal inconsistency in using the ideal solution theory to define the concentration at which major effects will be observed in the mechanical properties of the optimum gel. The inconsistency derives from the fact that the intrinsic viscosity relates to the conformation of chains at infinite dilution, whereas in the recovered gel the polymer chains have in principle gone through a theta condition.

Since there is no obvious theoretical approach to obtain the critical concentration for a dynamic situation such as exists in gel formation, we have used an empirical correlation between the drawability of the optimum gel and the characteristics of the dilute polymer solution. Such a correlation can be made by using the reduced viscosity-concentration relationship shown in Figures 1 and 2.

As can be seen in Figures 1 and 2, the plot can be classified into three reduced viscosity regimes. In the lower concentration regimes, the experimental points can be represented as two straight lines over two concentration regimes from 0 to $0.05 \mathrm{~g} / 100 \mathrm{ml}$ and from 0.05 to $0.1 \mathrm{~g} / 100 \mathrm{ml}$. In two regions, it is relatively simple to fit a tangent to the data. On the other hand, in the concentration regime higher than $0.6 \mathrm{~g} / 100 \mathrm{ml}$, the plots can be represented as a straight line roughly. Namely, in the regime of high concentration dependence, the continuously increasing gradient makes it difficult to fit the data with a single linear segment. Depending on how this is done, the intersection of the two linear segments could lie around $0.5 \mathrm{~g} / 100 \mathrm{ml}$. In this process, a line over the concentration range from 0.05 to $0.1 \mathrm{~g} / 100 \mathrm{ml}$ was selected to determine the intersection. As was already discussed for ultra-high molecular weight polyethylene, ${ }^{8-10}$ this seems to provide the most suitable level of chain segments to obtain fibers or films with maximum drawability associated with maxi- 

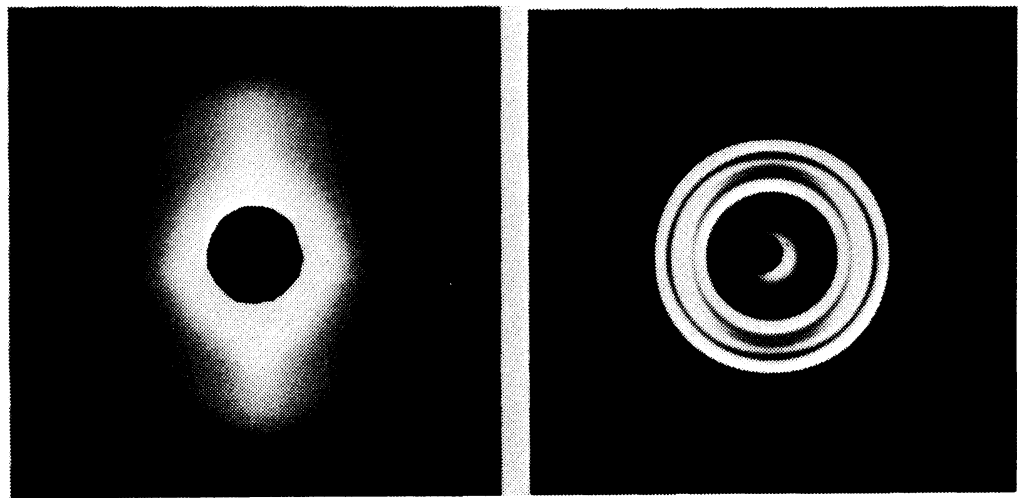

(a) $135^{\circ} \mathrm{C}$
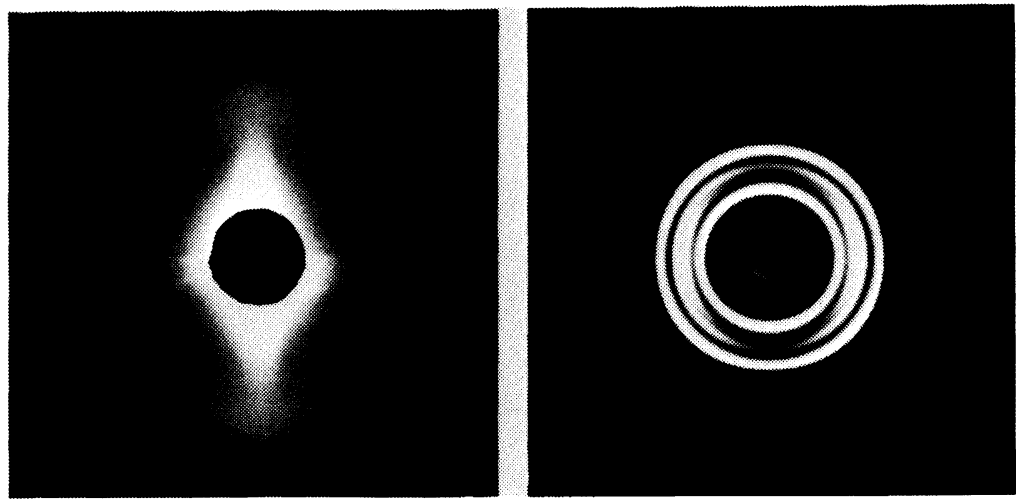

(b) $150^{\circ} \mathrm{C}$
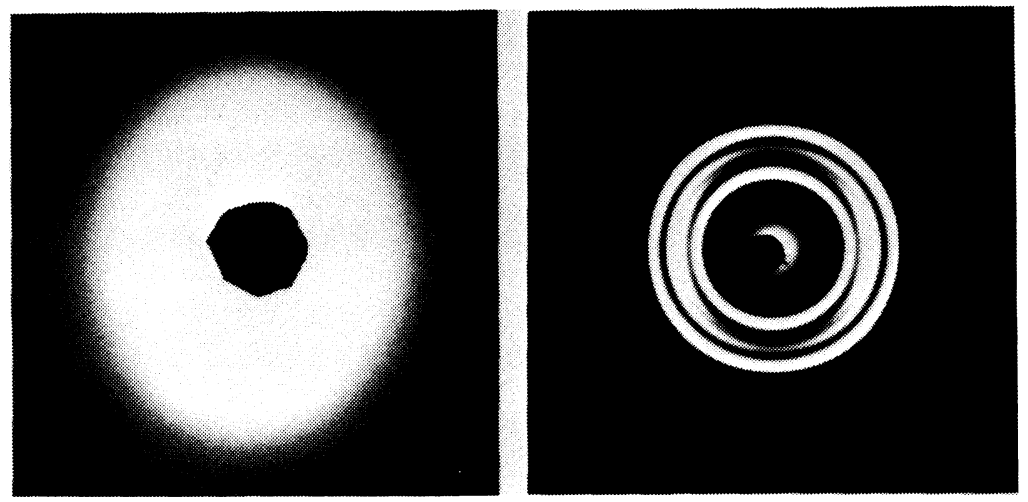

(c) $170^{\circ} \mathrm{C}$

Figure 3. SAXS and WAXD patterns (end view) from original dried gel films prepared by dissolution at temperatures 135,150 , and $170^{\circ} \mathrm{C}$.

mum modulus and strength.

Furthermore, it should be noted that the reduced viscosity for the polypropylene in Figure 1 is about one thirteenth that for the polyethylene used at the same concentration in the previous work, ${ }^{8}$ although the chain length of the former is about one-fifth that of the latter. Accordingly, this leads to the expecta- 
tion that the size or extension in space of polyethylene chains is much larger than that for polypropylene even if the molecular chain lengths for both the polymers are equal.

Figure 3 shows SAXS and WAXD patterns (end view) from original dried gel films prepared by dissolution at the temperatures 135,150 , and $170^{\circ} \mathrm{C}$. The WAXD patterns exhibit the preferential orientation of the crystal $c$-axes perpendicular to the film surface. The SAXS patterns of the dry gels prepared by dissolving at 135 and $150^{\circ} \mathrm{C}$ show distinct scattering maxima similar to the pattern from original dry gel films of polyethylene. ${ }^{17}$ Both the WAXD and SAXS patterns indicate that the structure of the dried gel films are similar to that of single crystal mats. The SAXS pattern of the dry gel film prepared by dissolving at $170^{\circ} \mathrm{C}$ shows diffuse scattering indicating large orientational fluctuation and fluctuation in lamellar size. These results of dried gel films indicate that within the lamellar crystals constructing the dry gel, the crystal $c$ axes are oriented perpendicular to the large flat faces. Thus, when the as-cast polypropylene gel films are dried by slow evaporation of solvent, the constituent lamellar crystals become oriented parallel to the film surface.

In order to facilitate understanding the morphology of dry gel films SAXS intensity distributions for three kinds of specimens were measured in the meridional direction with a PSPC system. These are shown in Figure 4. The distributions for the specimens prepared by dissolving at 135 and $150^{\circ} \mathrm{C}$ show the second order scattering maxima showing long periods to be 95 and $105 \AA$, respectively. In contrast, the profile of the intensity distribution for the specimen prepared by dissolving at $170^{\circ} \mathrm{C}$ shows a monotonical curve. Comparing these three profiles, it is obvious that $150^{\circ} \mathrm{C}$ is the most suitable temperature to prepare dried gel films composed of crystal lamellae that are highly oriented with their large flat faces parallel to the film surface. This is consistent

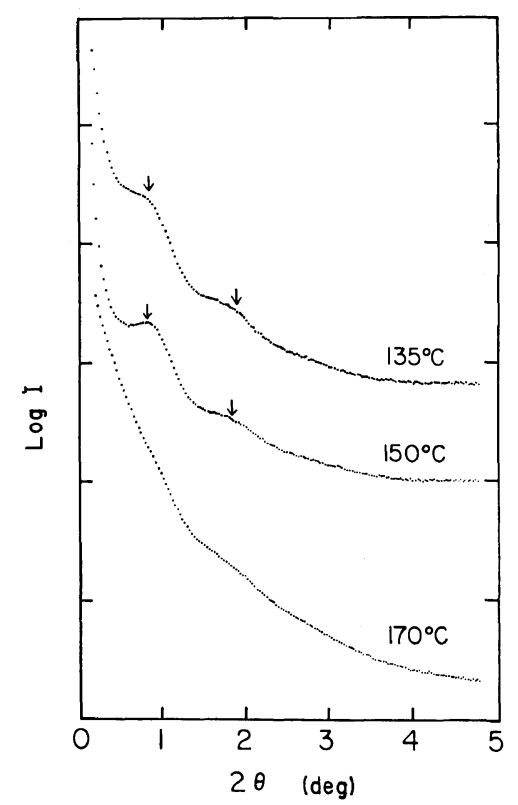

Figure 4. SAXS intensity distributions from undrawn dried gel films prepared by disolution at three temperatures 135,150 , and $170^{\circ} \mathrm{C}$.

with the result of viscosity measurement discussed before, since the degradation of molecular weight (in the case of $170^{\circ} \mathrm{C}$ ) as well as unsufficient dissolution (in the case of $135^{\circ} \mathrm{C}$ ) prevent preparing lamellae under gelation/ crystallization and then imperfect small lamellae were not sufficiently oriented parallel to the film surface. Thus all following experiments were carried out using the gels prepared by disolution at $150^{\circ} \mathrm{C}$.

Figure 5 shows WAXD patterns (through view) with increasing draw ratio $\lambda$. The decreasing angular spread of the strong equatorial (130), (040), and (110) reflections indicates an increase in the orientation of the crystal $c$-axes with respect to the stretching direction.

Figure 6 shows the variation of birefringence with draw ratio $\lambda$. The value increased with increasing $\lambda$ and this tendency became considerable up to $\lambda=20$ but was gradual with further elongation. This is consistent with the crystal orientation of the WAXD patterns in Figrue 5. Finally the value attained $27 \times$ 


\section{Ultradrawing of Polypropylene Films}
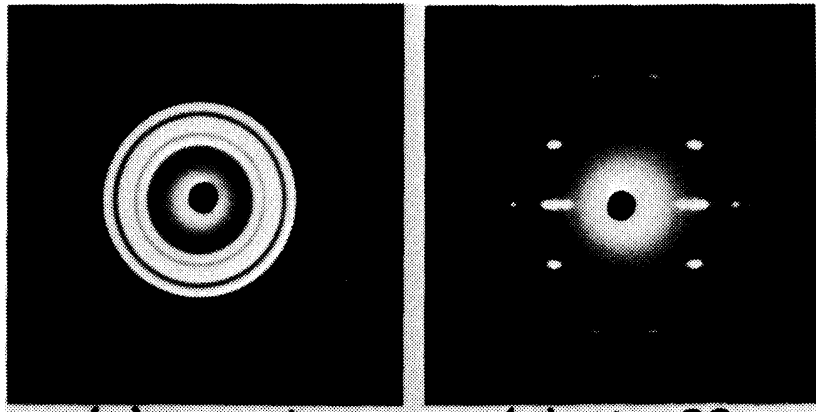

$$
\text { (a) } \lambda=1
$$

(e) $\lambda=20$
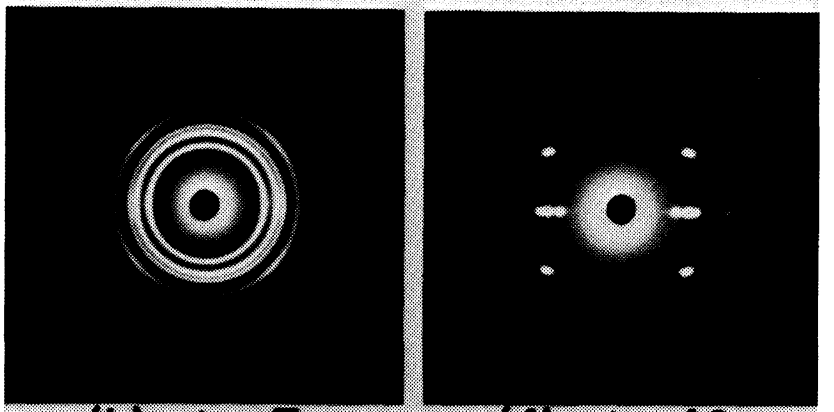

(b) $\lambda=3$
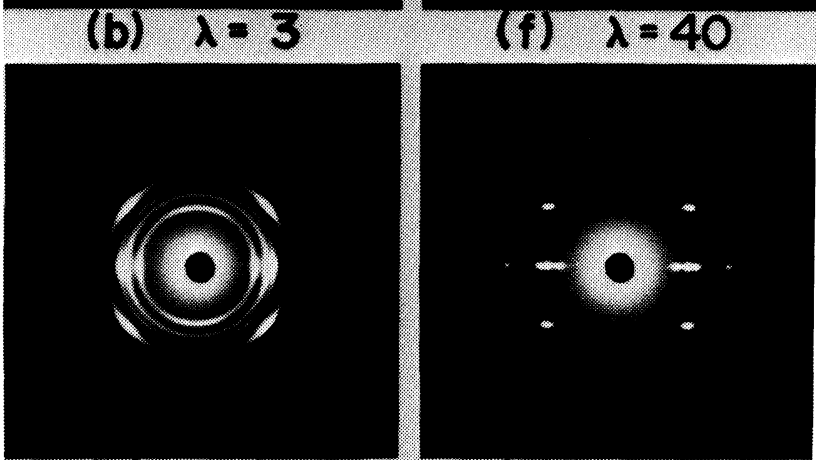

(c) $\lambda=5$

(g) $\lambda=60$

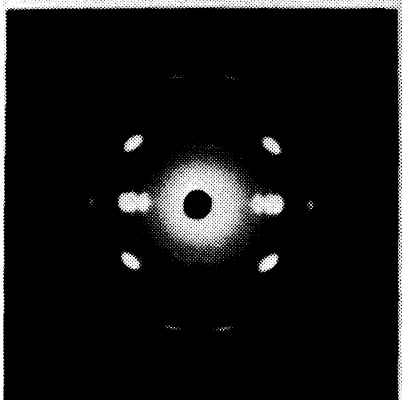

(d) $\lambda=10$

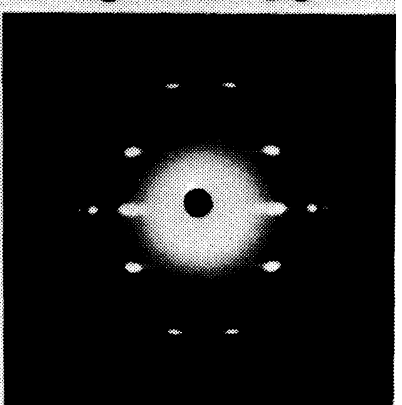

(h) $\lambda=100$

Figure 5. WAXD pattern (through view) of polypropylene dried gel films with increasing draw ratio $\lambda$. 
M. Matsuo, C. Sawatari, and T. Nakano

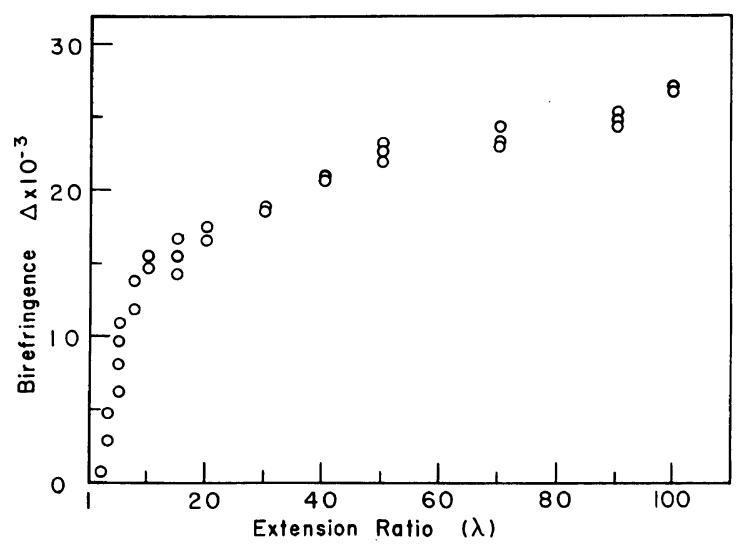

Figure 6. Birefringence of drawn polypropylene gel films as a function of the draw ratio $\lambda$.

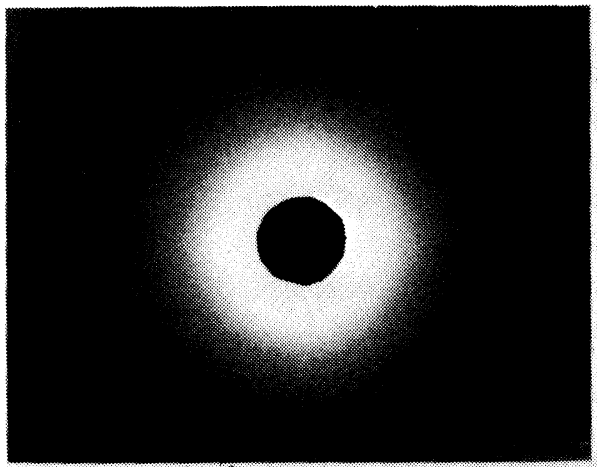

(a) $\lambda=1$

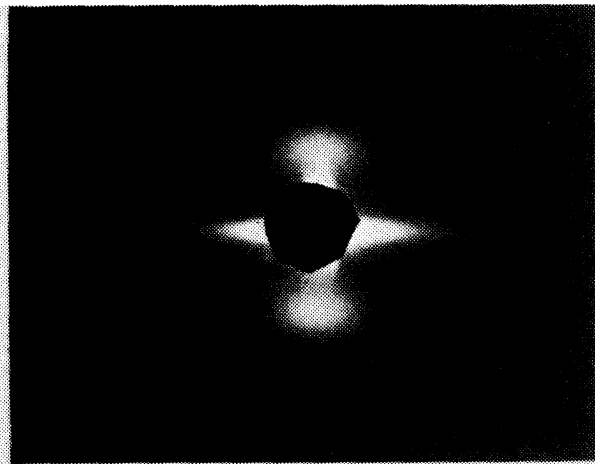

(c) $\lambda=10$

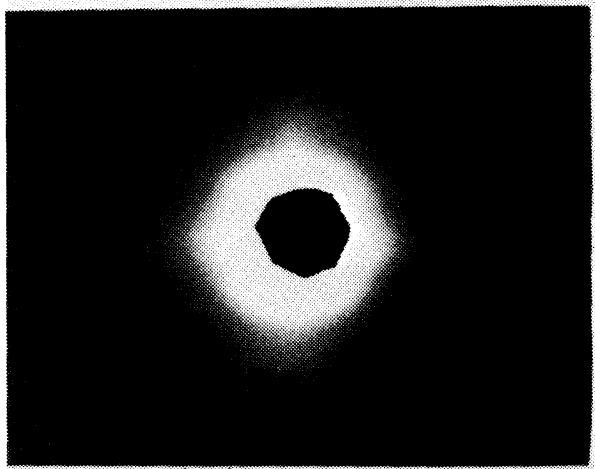

(b)

$\lambda=7$

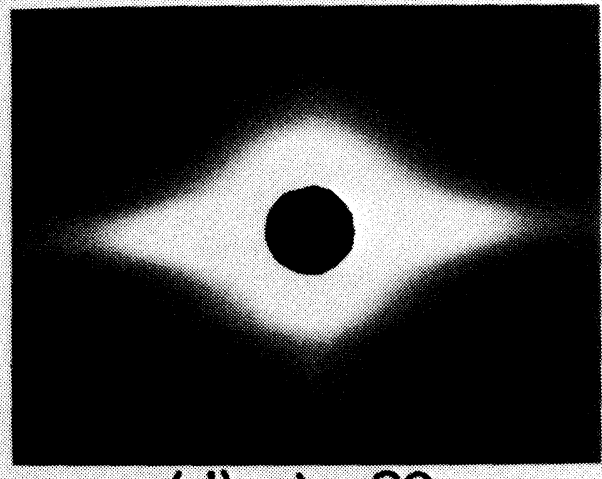

(d) $\lambda=20$

Figure 7. SAXS patterns (through view) of drawn polypropylene gel films as a functions of the draw ratio $\lambda$. The stretching direction is a vertical one.

$10^{-3}$ at $\lambda=100$. This value is the highest one reported for polypropylene and is rather close to $33.1 \times 10^{-3}$ corresponding to the intrinsic crystal birefringence estimated by Samuels using X-ray diffraction and the measurement of sonic modulus. ${ }^{16}$ 
Figure 7 shows SAXS patterns (through view) for the films that were drawn up to $\lambda=$ 20. The pattern for an original film shows a circular-type diffuse scattering characterizing random orientation of crystal lamellae with respect to the direction normal to the film surface. The patterns for drawn films show equatorial streaks that originate in scattering from elongated voids and the elongation of the streaks becomes considerable with increasing $\lambda$. Here it should be noted that the pattern at $\lambda=10$ shows the scattering maxima in the meridional direction indicating the existence of long period and the maxima disappeared at $\lambda=20$. The disappearance is probably due to the long period becoming longer with increasing $\lambda$ and the scattering maxima moving to the scattering center. With our X-ray in-
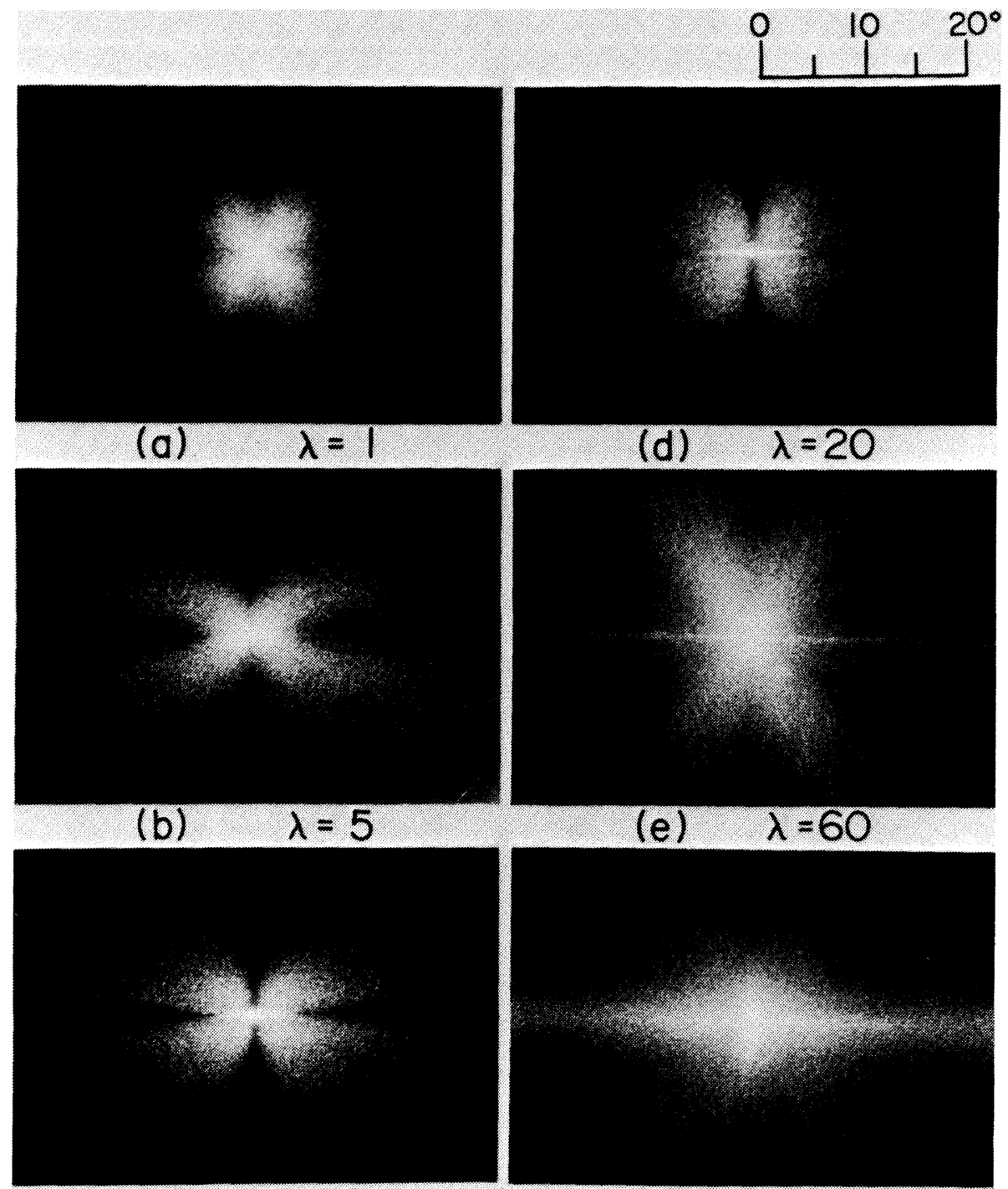

$$
\text { (c) } \quad \lambda=10
$$

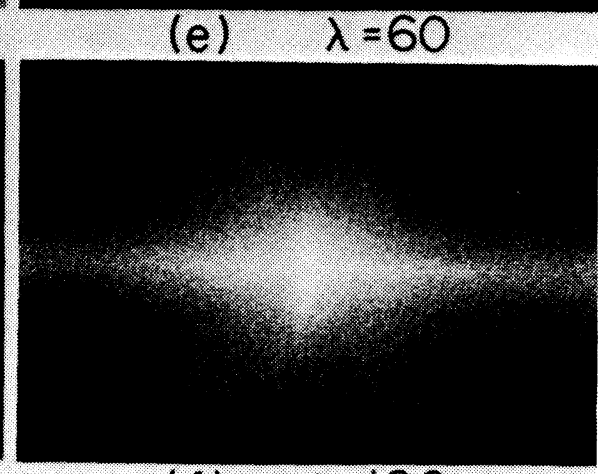

(f) $\quad \lambda=100$

Figure 8. SALS patterns from drawn polypropylene gel films under Hv polarization conditions. The stretching direction is vertical. 


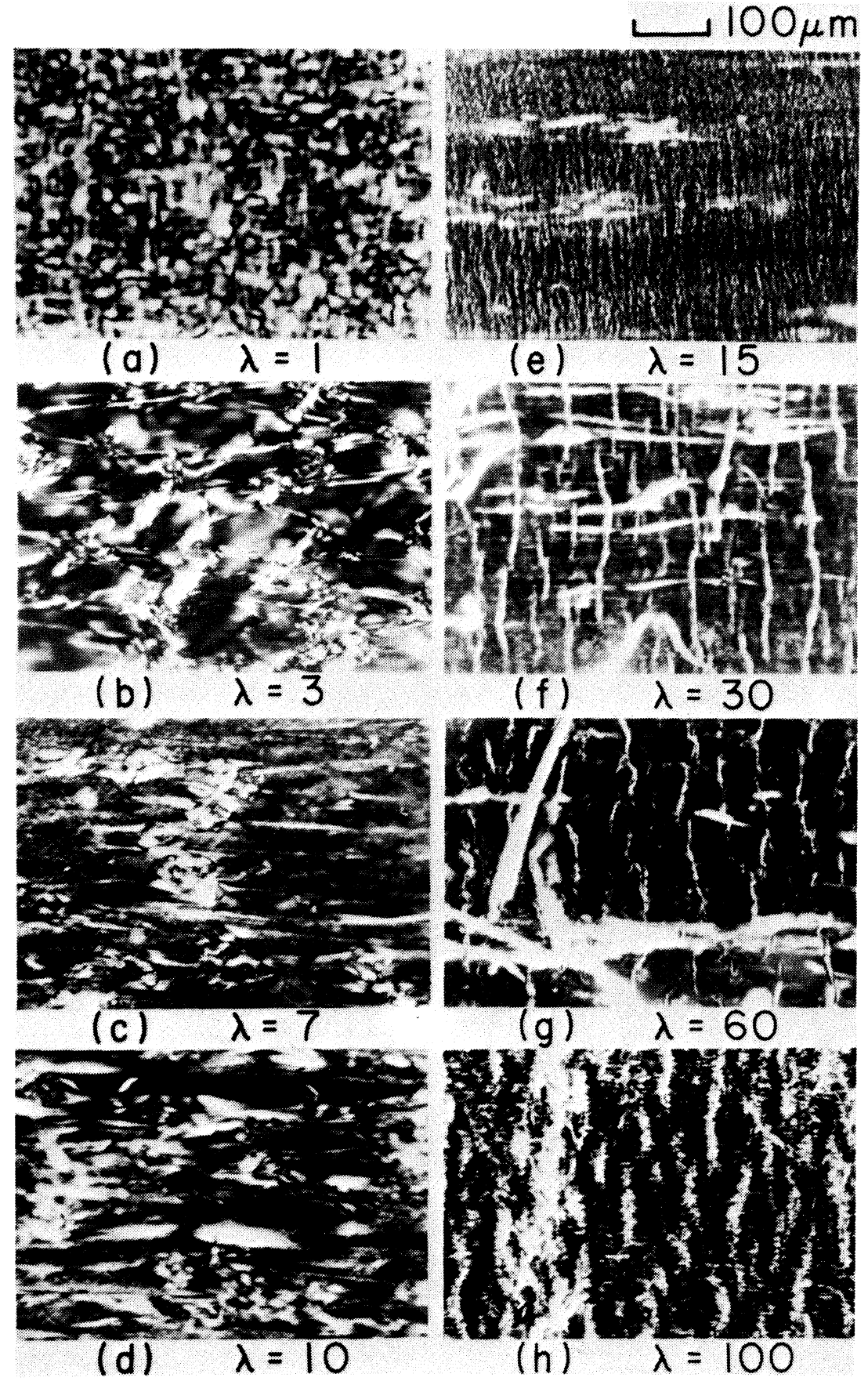

Figure 9. Polarized micrographs for drawn polypropylene gel films. The stretching direction is horizontal. 

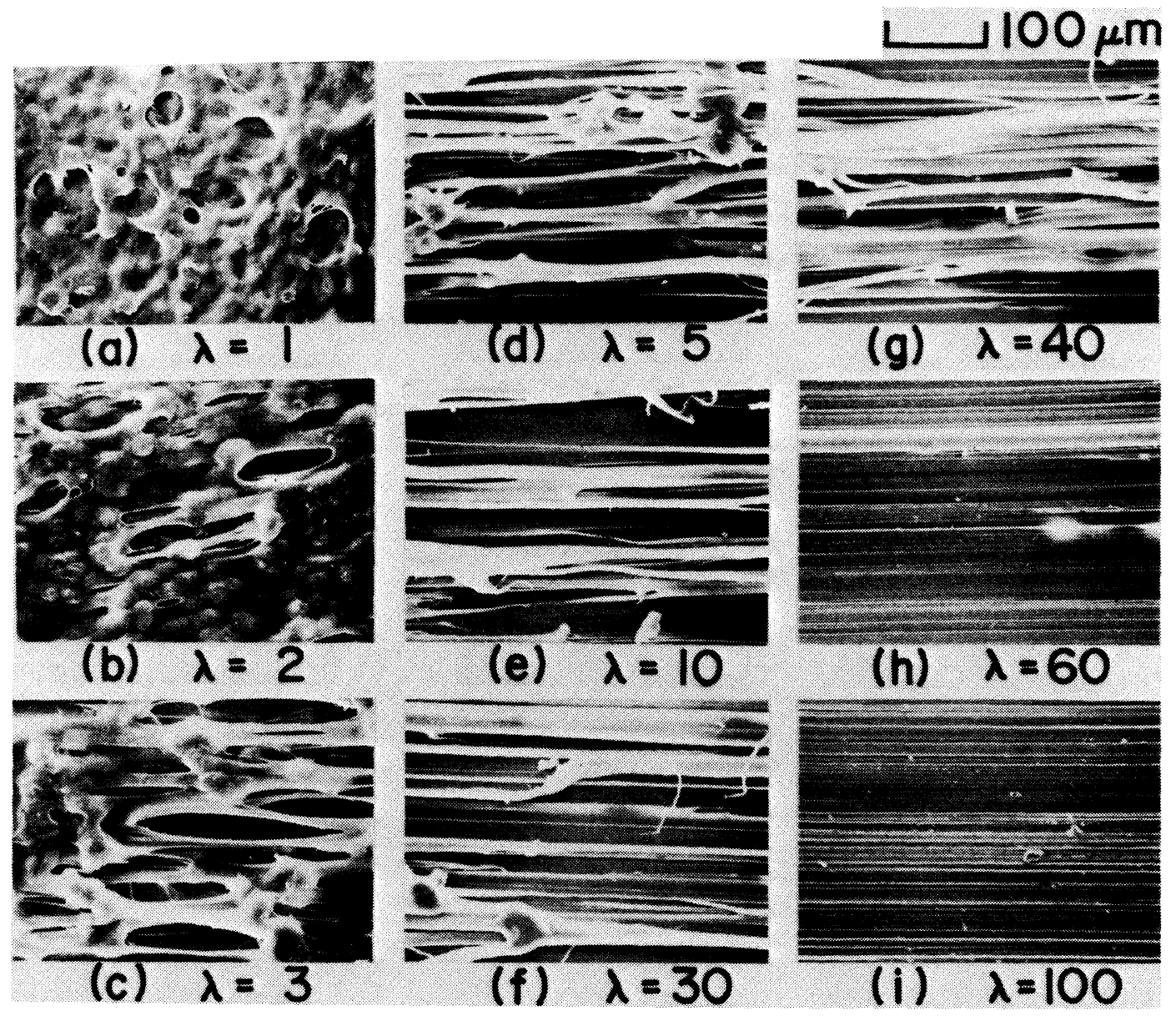

Figure 10. Scanning electron micrographs for drawn polypropylene gel films. The stretching direction is horizontal.

strument, unfortunately, it was impossible to detect the long period beyond $200 \AA$ because of the short distance between sample and detector.

Figure 8 shows $H_{v}$ light scattering patterns with increasing $\lambda$. Pattern (a) of the undrawn film displays lobes of a diffuse $X$ type. The intensity had a maximum in the scattering center and decreased monotonically with increasing scattering angle. These observations are indicative of scattering from rodlike textures. The existence of rodlike textures was already observed for polyethylene dried gel films as have been already ob- served for ultra-high molecular weight polyethylene gels. ${ }^{17,18}$ As for low molecular weight polyethylene, the dried gel cannot form a film and within the film there exist spherulitic textures. ${ }^{19}$ Judging from the relationship between the morphology of the superstructure and molecular weight for polyethylene dry gels, it may be postulated that the existence of rodlike textures within dry gel polypropylene films is also due to the ultra-high molecular weight used in the present work.

The scattering lobes up to $\lambda=10$ are extended in a horizontal direction, indicating the preferential orientation of rods in the stret- 
ching direction. In contrast, beyond $\lambda=20$, the scattering lobes are extended in a vertical direction. This tendency has been observed for the oriented crystallization process, and it was confirmed that the crystallinity increased with $\lambda$ as shown in Figure 11.

Figure 9 shows polarized micrographs with increasing draw ratio $\lambda$. The stretching direction is horizontal. A series of micrographs displays that there exist rodlike textures and the rods orient in the stretching direction, as confirmed by SAXS in Figure 7. At the draw ratio of $\lambda=15$, observation revealed that the drawn film consists of two alternating zones; unoriented domains (white parts in the micrographs) perpendicular in the stretching direction and oriented areas (dark parts in the micrographs). As the draw ratio increases, the oriented zones are enlarged considerably at the expense of the unoriented zones. Thus, the drawn process is nonuniform. At $\lambda=100$, the molecular orientation can be observed even in the unoriented zones observed for the specimen with $\lambda=60$, corresponding to the white parts in photograph (h).

Figure 10 shows the change in the appearance of a specimen with increase of draw ratio $\lambda$ under SEM. The textures in an undeformed state show spongy-like-tissue comprising interconnected lamellar crystals. With increas-

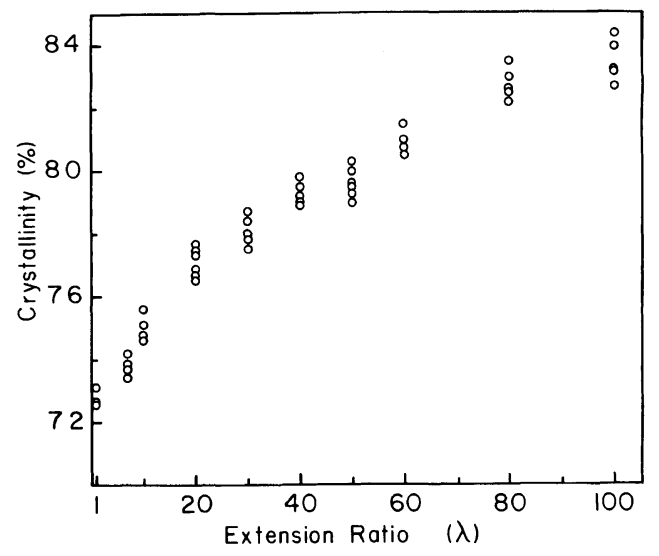

Figure 11. Crystallinity of drawn polypropylene gel films as a function of the draw ratio $\lambda$. ing $\lambda$, the width of the texture decreases and the resultant texture is similar to the fibrillar one. Finally, the fibrillar texture seems to be disruptively deformed into fine filaments. The filaments are also highly oriented parallel to the drawing direction. As illustrated in a series of photographs, this tendency becomes clear beyond $\lambda=20$. Photograph (d) indicates that the cross-linking loci appeared to consist of fibrillar shish-kabab-like crystals arranged in the drawn direction.

Figure 11 shows the change in crystallinity with draw ratio $\lambda$. The value increases with increasing $\lambda$ and attains $84 \%$ at $\lambda=100$. The maximum crystallinity of polypropylene is lower than that of polyethylene $(97-98 \%)$. This difference is uncertain but probably due to the conformation of molecular chains within crystallites.

Figure 12 shows the change in the profile of the DSC curves with increasing draw ratio $\lambda$. To check the reappearance of the profiles, DSC measurements on a given draw ratio were carried out several times. Unfortunately, it was found that the reappearance was much

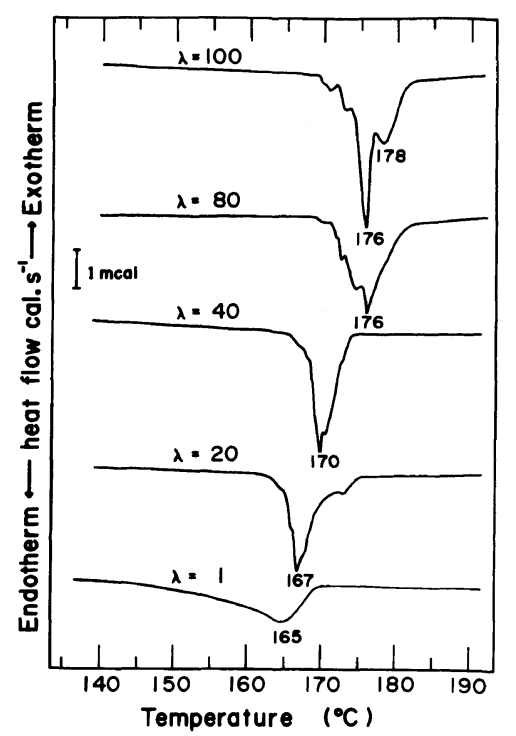

Figure 12. DSC curves of polypropylene gel films drawn for various draw ratios $\lambda$. Scan speed, $10 \mathrm{~K} /$ $\min ^{-1}$ 
worse than the results of ultradrawn polyethylene,$^{8}$ because of the shrinkage of the drawn specimens during heating. Then the representative results are shown in Figure 12. The profile shows a large main peak with small shoulders. At $\lambda=100$, the shoulder becomes the second peak. The main peak position shifts to higher temperature with increasing $\lambda$. The second peak at $\lambda=100$ attains $178^{\circ} \mathrm{C}$ which is close to the equilibrium melting point $\left(186 \pm 2^{\circ} \mathrm{C}\right){ }^{20}$ However, the measurable melting point is lower than the equilibrium value. This indicates that ultradrawn polypropylene is independent of superheating phenomenon associated with that polymer
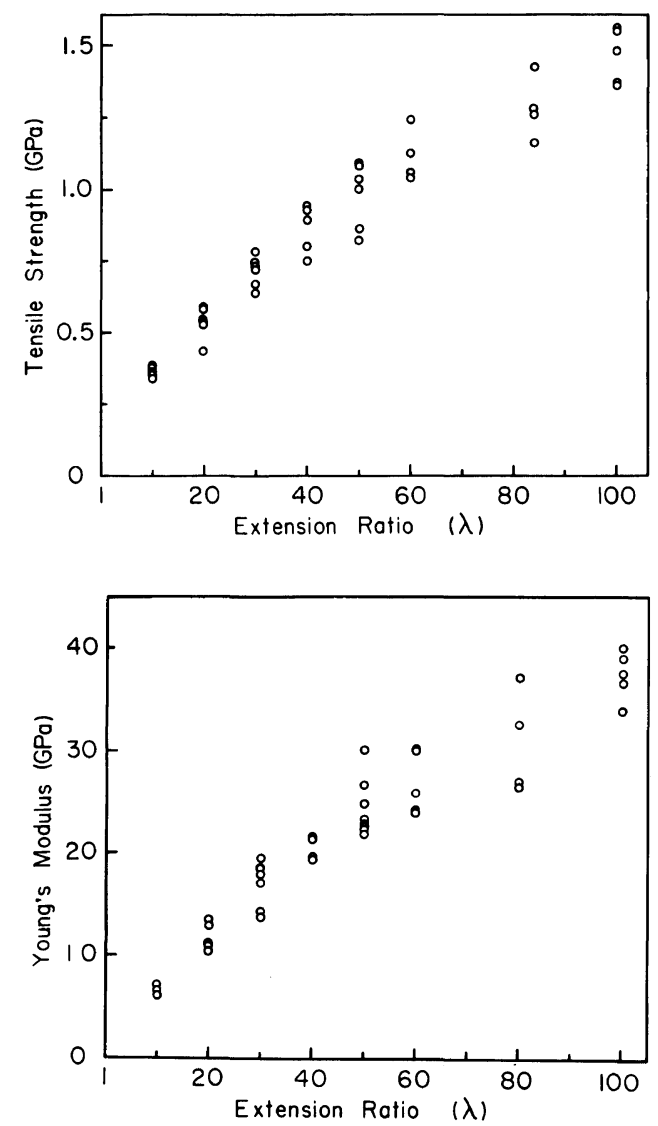

Figure 13. Mechanical properties of drawn polypropylene gel films. (a) Relationship between tensile strength and draw ratio $\lambda$. (b) Relationship between the Young's modulus and draw ratio $\lambda$. chains in the melt retaining the extended chain arrangement and therefore the entropy of fushion would obviously be smaller than the value obtained from random coils in the melt. Such a superheating phenomenon was observed for ultradrawn polyethylene. ${ }^{8}$ This difference between ultradrawn polyethylene and polypropylene is an unresolved problem.

Figure 13 shows tensile strength and the Young's modulus as a function of the draw ratio $\lambda$. The strain speed was selected in the range between 25 and $125 \% \mathrm{~min}^{-1}$. The Young's modulus and tensile strength increase linearly with increasing $\lambda$ and assure maximum values of 40.4 and $1.56 \mathrm{GPa}$, respectively. These values are among the highest reported ones for polypropylene at room temperature. The value of the tensile strength is much higher than $1.03 \mathrm{GPa}$ reported by Peguy and Manley ${ }^{12}$ and also higher than the theoretical value $1.23 \mathrm{GPa}$ reported by Samuels. ${ }^{21}$ The observed Young's modulus is comparable to the modulus of polypropylene crystal lattice in the direction of the chain axis as determined by X-ray diffraction. The crystal lattice modulus was estimated by Sakurada et al. using the apparent crystal lattice modulus of the (113) crystal plane. ${ }^{22}$ The apparent value was $41.2 \mathrm{GPa}$ for monofilament and $47 \mathrm{GPa}$ for drawn film. Based on this value of filament, the real value of the crystal lattice modulus was evaluated to be $35 \mathrm{GPa}$ through differential calculus of crystal lattice unit. ${ }^{23}$ Unfortunately, their calculation contains a fundamental misunderstanding. According to our calculation method, ${ }^{24}$ the real crystal lattice modulus in the direction of the molecular chain can be estimated through linear transformation of the Cartesian coordinates accompanied by rotation of the coordinate axes of stress and strain. The value was in the range between 38.7 and 43.3 GPa. In this case, the Young's moduli of ultradrawn films with $\lambda=100$ are close to the theoretical values. Accordingly, 
it is of interest to produce bulk polypropylene whose the Young's modulus is almost close to the ultimate goal.

The tensile strength measured in this experiment is beyond the theoretical value (1.23 $\mathrm{GPa}) .^{21}$ This indicates that further modification for the theoretical treatment must be carried out.

Now, we shall compare the results in Figure 13 with those of Peguy and Manley ${ }^{12}$ in terms of the relationship between the Young's modulus and draw ratio $\lambda$. The present values at $\lambda=20$ and 40 are about 10 and $20 \mathrm{GPa}$, respectively and their corresponding values are about 20 and $30 \mathrm{GPa}$, respectively. However, the highest draw ratio in their experiment was 47 times. This is probably because the present polypropylene gels were prepared from a sample of higher molecular weight than that of their sample $\left(\bar{M}_{v}=3.4 \times 10^{6}\right)$ and it may be expected to obtain a less oriented material for the same draw ratio but to enable elongation up to $\lambda=100$.

Figures 14 and 15 show temperature dependence of the real and imaginary parts of the complex dynamic tensile modulus of drawn films, respectively. All the specimens were annealed for $1 \mathrm{~h}$ at $150^{\circ} \mathrm{C}$ prior to the measurement in order to discuss the profile of the storage and loss moduli without considering effects on thermal crystallization only in relation to draw ratio. The dynamic modulus was measurable beyond $150^{\circ} \mathrm{C}$ for a specimen drawn beyond $\lambda=20$ but the undrawn film was broken by partial melting around $80^{\circ} \mathrm{C}$. The magnitude of storage modulus $E^{\prime}$ increases as the temperature decreases. The values of $E^{\prime}$ at $20^{\circ} \mathrm{C}$ are consistent with the Young's moduli in Figure 13.

Draw ratio causes a significant change in the profile of temperature-dependence of $E^{\prime \prime}$. The relationship between $E^{\prime \prime}$ and $T$ exhibited $\alpha, \beta$, and $\gamma$ peaks in order of decreasing temperature. ${ }^{25-27}$ The analysis of the three mechanisms has been reported as follows: The $\alpha$ mechanism is due to the crystal dispersions

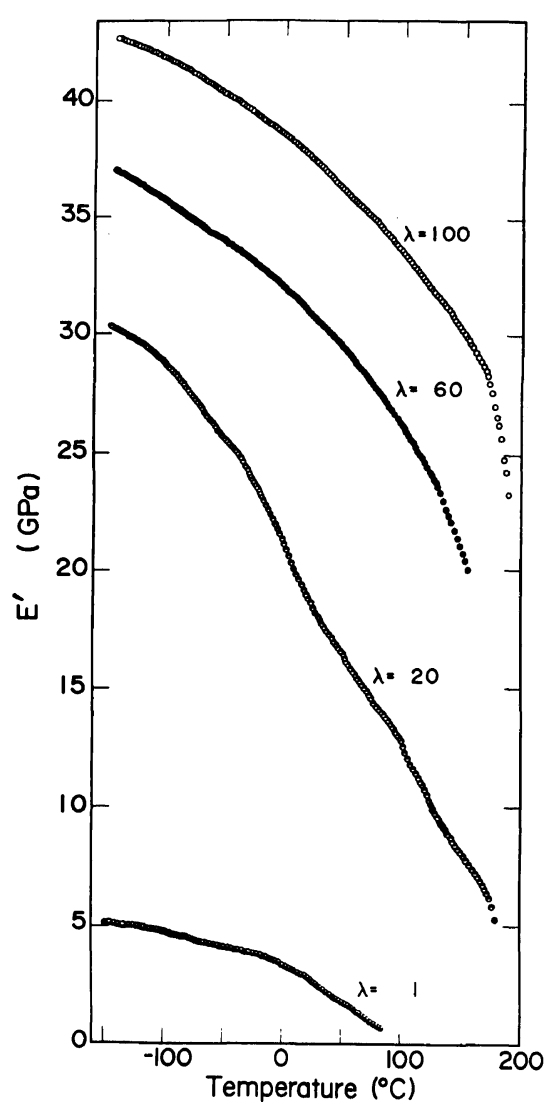

Figure 14. Storage modulus of drawn polypropylene films as a function of temperature.

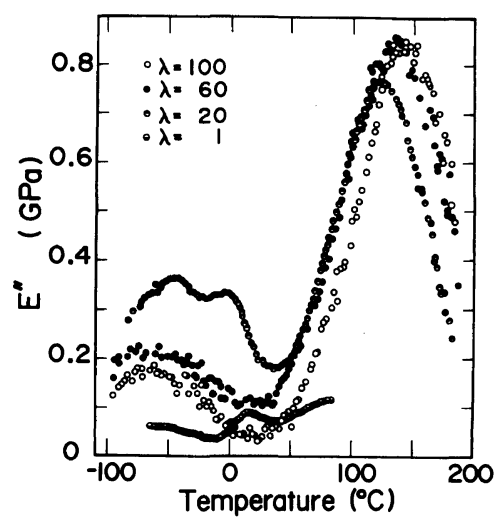

Figure 15. Loss moduli of drawn polypropylene films as functions of temperature.

associated with grain boundary phenomena arising from deformation and/or rotation of elastic grains within a viscoelastic amorphous 
medium as well as a crystal disordering transition due to onset of torsional and/or oscillation of polymer chains within the crystal lattice. The $\beta$ process corresponds to the transition from the glassy to the rubber-like state and the $\gamma$ process is usually assigned to the local mode relaxation in the amorphous phase.

As can be seen in Figure 15, the profile of original film shows the $\beta$ dispersion around $20^{\circ} \mathrm{C}$. This is quite consistent with the previous result for a solution crystallized sample. ${ }^{28}$ The $\beta$ peak for the specimen at $\lambda=20$ shifts to a lower temperature around $-5^{\circ} \mathrm{C}$. This indicates that with increasing $\lambda$, segmental motions in the amorphous phase become more pronounced, since the crystallinity increases and the amorphous regions behave somewhat like paracrystallites. However, this is essentially an unresolved problem. In addition to the peak, another peak can be observed around $-45^{\circ} \mathrm{C}$. This small peak becomes considerable at $\lambda=60$ and 100 , instead of the disappearance of the $\beta$ peak. This peak is probably attributed to the $\gamma$ dispersion. According to the report by Inamura et al., ${ }^{28}$ the $\gamma$ dispersion of solution-crystallized polypropylene film arises from crystal defects in the crystalline phase and local relaxation modes from twisting of the main chains in the amorphous phase. They pointed out that the dispersion peak around $-85^{\circ} \mathrm{C}$ corresponded to the defects but not the local relaxation mode. In such a case, crystal defects but not the local relaxation mode may be expected. This being so, it may be expected that the peak around $-70^{\circ} \mathrm{C}$ for the present sample at $\lambda=60$ corresponds to the dispersion associated with crystal dispersion, because of the high crystallinity above $82 \%$.

The peak associated with the $\alpha$ dispersion shifts to higher temperature with increasing $\lambda$ as has been observed generally for polyethylene. This is attributed to the decrease in the number of crystal defects through elongation. This detailed phenomena will be dis- cussed elsewhere in terms of frequencydependence of $E^{\prime \prime}$ against various temperatures. $^{29}$

\section{CONCLUSIONS}

The viscosity/concentration data presented for high molecular weight polypropylene $\left(\bar{M}_{w}=4.4 \times 10^{6}\right)$ indicates that there exists a critical concentration where an abrupt change occurs in the relationship between the viscosity and the concentration. The critical concentration provides a suitable level of entanglements through gelation/crystallization and the dry process.

The superstructure of a dried gel film was studied by SAXS, WAXD, SALS, SEM, birefringence, and polarized microscopy. Observations revealed the existence rodlike textures. The rod was composed of crystal lamellae highly oriented with their large flat faces parallel to the film surface. Furthermore, within the crystal lamellae the crystal $c$ axes are oriented perpendicular to the large flat faces in a manner similar to the mats of single crystals.

The dried gel films were stretched to a maximum draw ratio of $\lambda=100$. For such drawn films with $\lambda=100$, the tensile strength and the Young's modulus attained maximum values of 1.56 and $40.4 \mathrm{GPa}$, respectively, at $20^{\circ} \mathrm{C}$. These are among the highest values reported for polypropylene. The value of tensile strength is higher than that of theoretical values. $^{21}$ and the Young's modulus is close to the theoretical value ascertained by $\mathrm{X}$-ray diffraction. ${ }^{24}$

\section{REFERENCES}

1. S. Kojima, C. R. Desper, and R. S. Porter, J. Polym. Sci., Polym. Phys. Ed., 16, 1721 (1978).

2. J. Clements, G. Capaccio, and I. M. Ward, J. Polym. Sci., Polym. Phys. Ed., 17, 693 (1979).

3. J. Smook, J. C. Torfs, P. F. van Hulten, and A. J Pennings, Polym. Bull., 2, 293 (1980).

4. P. J. Barham and A. Keller, J. Mater. Sci., 15, 2229 (1980). 
5. P. Smith and P. J. Lemstra, J. Mater. Sci., 15, 505 (1980).

6. P. Smith, P. J. Lemstra, and H. C. Booij, J. Polym. Sci., Polym. Phys. Ed., 19, 877 (1981).

7. P. Smith, P. J. Lemstra, J. P. L. Pippers, and A. M. Kiel, Colloid Polym. Sci., 258, 1070 (1981).

8. C. Sawatari and M. Matsuo, Colloid Polym. Sci., 263, 783 (1985).

9. M. Matsuo, Nihon Reoroji Gakkaishi, 13, 4 (1985).

10. M. Matsuo, K. Inoue, and N. Abumiya, Sen- $i$ Gakkaishi, 40, 275 (1984).

11. M. Matsuo and C. Sawatari, Macromolecules, 19, 2036 (1986).

12. A. Peguy and R. S. T. Manley, Polym. Commun., 25, 39 (1984).

13. T. Kanamoto, A. Tsuruta, K. Tanaka, and M. Takeda, Polym. Prepr. Jpn., 33, 779 (1984).

14. G. Natta and P. Corradini, Nuovo Cimento, Suppl., 15, 40 (1960).

15. W. W. Graessley, Adv. Polym. Sci., 16, 3 (1974).

16. R. J. Samuels, J. Polym. Sci., A, 3, 1741 (1965).

17. M. Matsuo and R. S. T. Manley, Macromolecules, 15, 985 (1982).
18. M. Matsuo and R. S. T. Manley, Macromolecules, 16, 1500 (1983).

19. C. Sawatari, T. Okumura, and M. Matsuo, Polym. J., 18, 741 (1986).

20. W. R. Krigbaum, and I. Uematsuo, J. Polym. Sci A, 3, 767 (1965).

21. R. J. Samuels, J. Macromol. Sci.-Phys., B4, 701 (1970).

22. I. Sakurada, T. Ito, and K. Nakamae, J. Polym. Sci., C, 15, 75 (1966)

23. I. Sakurada, and K. Kaji, J. Polym. Sci., 31, 57 (1970).

24. C. Sawatari and M. Matsuo, Macromolecules in press.

25. S. Iwayanagi and I. Sakurai, J. Polym. Sci., C, 14, 29 (1966).

26. K. M. Sinnot, J. Polym. Sci., C, 14, 141 (1966).

27. M. Takayanagi and T. Matsuo, J. Macromol. Sci.Phys., B1, 407 (1967).

28. I. Inamura, H. Ochiai, and H. Yamamura, J. Polym. Sci., Polym. Phys. Ed., 12, 2267 (1974).

29. M. Matsuo and C. Sawatari, in preparation. 